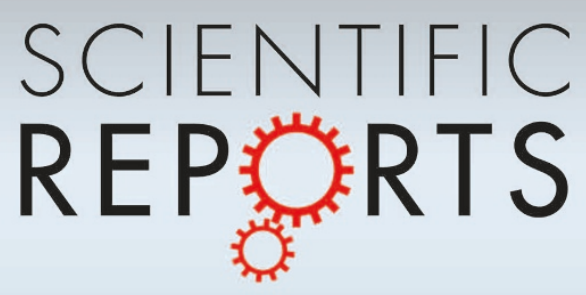

OPEN

SUBJECT AREAS:

PLANT PHYSIOLOGY

PLANT MOLECULAR BIOLOGY

PLANT BREEDING

PLANT TRANSPORTERS

Received

19 December 2014

Accepted

9 March 2015

Published

29 April 2015

Correspondence and requests for materials should be addressed to M.Z. (zhangmy@scbg. ac.cn)

* These authors contributed equally to this work.

\section{Disruption of the rice nitrate transporter OsNPF2.2 hinders root-to-shoot nitrate transport and vascular development}

\author{
Yuge Li'*, Jie Ouyang ${ }^{2 *}$, Ya-Yun Wang ${ }^{3}$, Rui Hu ${ }^{1,4}$, Kuaifei Xia ${ }^{1}$, Jun Duan' ${ }^{1}$, Yaqin Wang ${ }^{5}$, Yi-Fang Tsay ${ }^{6}$ \\ \& Mingyong Zhang ${ }^{1}$
}

\begin{abstract}
${ }^{1}$ Key Laboratory of South China Agricultural Plant Molecular Analysis and Genetic Improvement \& Guangdong Provincial Key Laboratory of Applied Botany, South China Botanical Garden, Chinese Academy of Sciences, Guangzhou 510650, China, ${ }^{2}$ Rice Institute, Chongqing Academy of Agricultural Sciences, Chongqing 401329, China, ${ }^{3}$ Department of Life Science, National Taiwan University, Taipei, Taiwan, ${ }^{4}$ University of Chinese Academy of Sciences, Beijing 100049, China, ${ }^{5}$ Guangdong Key Lab of Biotechnology for Plant Development, College of Life Science, South China Normal University, Guangzhou 510631, China, Institute of Molecular Biology, Academia Sinica, Taipei, Taiwan.
\end{abstract}

Plants have evolved to express some members of the nitrate transporter 1/peptide transporter family (NPF) to uptake and transport nitrate. However, little is known of the physiological and functional roles of this family in rice (Oryza sativa L.). Here, we characterized the vascular specific transporter OsNPF2.2. Functional analysis using cDNA-injected Xenopus laevis oocytes revealed that OsNPF2.2 is a low-affinity, $\mathrm{pH}$-dependent nitrate transporter. Use of a green fluorescent protein tagged OsNPF2.2 showed that the transporter is located in the plasma membrane in the rice protoplast. Expression analysis showed that OsNPF2.2 is nitrate inducible and is mainly expressed in parenchyma cells around the xylem. Disruption of OsNPF2.2 increased nitrate concentration in the shoot xylem exudate when nitrate was supplied after a deprivation period; this result suggests that OsNPF2.2 may participate in unloading nitrate from the xylem. Under steady-state nitrate supply, the osnpf 2.2 mutants maintained high levels of nitrate in the roots and low shoot:root nitrate ratios; this observation suggests that OsNPF2.2 is involved in root-to-shoot nitrate transport. Mutation of OsNPF2.2 also caused abnormal vasculature and retarded plant growth and development. Our findings demonstrate that OsNPF2.2 can unload nitrate from the xylem to affect the root-to-shoot nitrate transport and plant development.

$\mathrm{N}$ itrogen $(\mathrm{N})$ is a primary nutritional element for plant growth and development. $\mathrm{N}$ is also a major limiting factor for crop yield ${ }^{1-2}$. However, if $\mathrm{N}$ fertilizers added to soils are not absorbed efficiently by plants, the fertilizers can cause serious environmental pollution. Nitrate $\left(\mathrm{NO}_{3}{ }^{-}\right)$and ammonium $\left(\mathrm{NH}_{4}{ }^{+}\right)$are two major $\mathrm{N}$ sources for higher plants. Like most upland plants, lowland rice uses nitrate and ammonium as its major $\mathrm{N}$ sources $^{3}$. In rice, nitrate acquisition is not only of high capacity and efficiency but is also considered superior to ammonium acquisition ${ }^{4}$. Furthermore, the growth and yield of rice is superior on mixtures of nitrate and ammonium than with provision of either $\mathrm{N}$ source alone ${ }^{3}$. Under cultivation, the proportion of available nitrate in soils increases, and $\mathrm{NO}_{3}{ }^{-}-\mathrm{N}$ becomes the dominant form instead of $\mathrm{NH}_{4}{ }^{+}-\mathrm{N}$, which is traditionally assumed to be the preferred $\mathrm{N}$ source for paddy rice ${ }^{5}$. Nitrate and ammonium concentrations range from less than $100 \mu \mathrm{M}$ to more than $10 \mathrm{mM}$ in soil solutions, so plant roots have uptake systems for both $\mathrm{NO}_{3}{ }^{-}$and $\mathrm{NH}_{4}{ }^{+}$, with different affinities $^{6,7}$.

Nitrate uptake and allocation are key factors in efficient $\mathrm{N}$ utilization for higher plants ${ }^{2,6}$. Membrane-bound transporters are required for nitrate uptake from the soil and for inter- and intracellular movement of nitrate inside the plants ${ }^{6}$. In Arabidopsis, at least five gene families are involved in the uptake, allocation, and sensing of nitrate ${ }^{1,6,8,9}$. These include the NPF (previously NRT1/PTR) family ${ }^{10}$ for low-affinity transport of nitrate or di/ tripeptides, the NRT2 family for high-affinity nitrate transport, the CLC family for chloride channels, the SLAC1/ SLAH family which contains the gene encoding slow anion channel-associated 1 homolog 3 , and the ALMT family for aluminum-activated malate transport.

Only a small proportion of the $53 \mathrm{NPF}$ genes in Arabidopsis have been functionally characterized for nitrate transport activity ${ }^{1,10}$. Some Arabidopsis NPFs are also able to transport other substrates, such as auxin, abscisic 
acid, and glucosinolates ${ }^{11-13}$. Most of the NPF nitrate transporters are low-affinity nitrate transporters ${ }^{1}$; only AtNPF6.3 (AtNRT1.1, CHL1) ${ }^{14,15}$ and MtNPF6.8 (MtNRT1.3) ${ }^{16}$ are dual-affinity nitrate transporters.

Nitrate is taken up by roots from soil and transported to shoots and seeds for storage and/or further assimilation ${ }^{1}$. AtNPF6.3 is involved in the uptake of nitrate from the soil ${ }^{14,17}$ and as a bidirectional transporter in the translocation of nitrate from the roots to the shoots $^{18}$. AtNPF7.3 (AtNRT1.5) is responsible for loading nitrate into the xylem for root-to-shoot nitrate transport ${ }^{19}$. AtNPF7.2 (AtNRT1.8) and AtNPF2.9 (AtNRT1.9) are involved in regulating root-to-shoot nitrate translocation of xylem and phloem respectively ${ }^{20,21}$. AtNPF6.2 (AtNRT1.4) regulates leaf nitrate homeostasis and leaf development, and AtNPF2.13 (AtNRT1.7) mediates phloem loading to allocate nitrate from older to younger leaves ${ }^{22,23}$. AtNPF2.12 (AtNRT1.6) is involved in delivering nitrate to developing seeds ${ }^{24}$.

AtNPF6.3 also functions as a nitrate sensor that regulates transcription ${ }^{25}$ and participates in promoting lateral root elongation in $\mathrm{NO}_{3}{ }^{-}$rich medium ${ }^{26}$. In the absence of nitrate, AtNPF6.3 facilitates the uptake of the phytohormone auxin ${ }^{13}$. These studies showed that nitrate-regulated AtNPF6.3-dependent auxin transport is responsible for nitrate-promoted lateral root elongation. The high-affinity uptake complex NRT2.1-NAR2.1 also participates in regulating lateral root development ${ }^{27}$; the regulation of lateral root growth by NRT2.1 and NAR2.1 is independent of their uptake function ${ }^{28}$.

Rice is a main cereal crop that provides the staple food for more than half of the world's population. However, the efficiency of nitrogen utilization by rice is lower than that for other $\operatorname{crops}^{29}$, and rice varieties show genetic variation with respect to the level of nitrogen fertilization required ${ }^{30}$. Most of our knowledge about nitrate uptake and translocation is from the study of Arabidopsis, and little is known about these processes in rice ${ }^{1,2}$. Therefore, an understanding of the molecular mechanisms of nitrate uptake and translocation in rice is very important for improving the efficiency of nitrogen use in rice. In the rice genome, there are more than 93 NPF genes ${ }^{10,31,32}$, and 5 NRT2 genes $^{33,34}$ have been predicted. However, to date, only six members of the rice NPF gene family have been studied, and only OsNPF8.9 $(\mathrm{OsNRT} 1)^{35}$ and OsNPF $2.4^{36}$ have been functionally demonstrated to transport nitrate. Here, we characterized the rice NPF family member OsNPF2.2 and show that it is a low-affinity nitrate transporter that participates in unloading nitrate from the xylem and influences root-to-shoot nitrate transport and plant development.

\section{Results}

OsNPF2.2 is a low-affinity nitrate transporter. Rice OsNPF2.2 (LOC_Os12g44100) is classified into the NPF2 subfamily of the $N P F$ family $^{10}$. It's mRNA (accession no. AK068351) is predicted to encode a 589 amino-acid protein that has a high degree of homology with low-affinity nitrate transporters. The OsNPF2.2 protein is $58 \%, 45 \%, 41 \%$, and $47 \%$ homologous to OsNPF2.4, AtNPF2.13, AtNPF2.12, and AtNPF2.9, respectively, all of which are involved in nitrate transport, and $48 \%$ and $50 \%$ homologous to AtNPF2.10 (AtGTR1) and AtNPF2.11 (AtGTR2), respectively, both of which are involved in glucosinolate transport. It contains 12 putative transmembrane domains (TMs) with a long hydrophilic loop between TM6 and TM7 (Supplementary Fig. S1); this structure is similar to the typical structure of NPF (NRT1/PTR) transporters ${ }^{37,38}$.

To determine whether OsNPF2.2 is a nitrate transporter, its cDNA was heterologously expressed in Xenopus laevis oocytes. We assayed high- and low- affinity nitrate transport activity with $250 \mu \mathrm{M}$ and $10 \mathrm{mM}^{15} \mathrm{NO}_{3}{ }^{-}$, respectively. As shown in Fig. 1a, as compared with water-injected oocytes, OsNPF2.2-injected oocytes at pH 5.5 showed significantly enhanced ${ }^{15} \mathrm{NO}_{3}{ }^{-}$uptake activity with $10 \mathrm{mM} \mathrm{NO}_{3}{ }^{-}$ after $1.5 \mathrm{~h}$ and $3 \mathrm{~h}$ of incubation. To confirm that OsNPF2.2 functioned as expected for a proton-coupled nitrate transporter, the
$\mathrm{NO}_{3}{ }^{-}$transport activity of OsNPF2.2-injected oocytes was measured at $\mathrm{pH} 5.5$ and $\mathrm{pH} 7.4$, respectively (Fig. 1b). The enhanced ${ }^{15} \mathrm{NO}_{3}{ }^{-}$ uptake activity of OsNPF2.2-injected oocytes was only detected at pH5.5. To determine the affinity of OsNPF2.2 for $\mathrm{NO}_{3}{ }^{-}$, the uptake activity of OsNPF2.2-injected oocytes at $\mathrm{pH} 5.5$ was measured using different concentrations, ranging from 0.5 to $30 \mathrm{mM}$, of ${ }^{15} \mathrm{~N}$-labeled $\mathrm{NO}_{3}{ }^{-}$, and the $\mathrm{K}_{\mathrm{m}}$ for $\mathrm{NO}_{3}{ }^{-}$, calculated by fitting the data to the Michaelis-Menten equation, was estimated as $\sim 16.6 \pm 12.9 \mathrm{mM}$ (Fig. 1c). The results from the functional studies in Xenopus oocytes indicate that OsNPF2.2 is a $\mathrm{pH}$-dependent, low-affinity nitrate transporter.

OsNPF2.2 is localized in the plasma membrane. To determine the subcellular location of OsNPF2.2, an OsNPF2.2-GFP fusion protein under the control of the CMV $35 S$ promoter $^{39}$ was transiently coexpressed with the plasma membrane marker pm-rk-mcherry ${ }^{40}$ in rice protoplasts. Unlike the signal for free GFP (Fig. 2f), which was found in the whole cell, the signal for the OsNPF2.2-GFP fusion protein (Fig. 2a) was localized in the plasma membrane and overlapped (Fig. 2d) with the signal for the marker pm-rk-cherry (Fig. 2b). Taken together with the observation that OsNPF2.2 has 12 TMs (Supplementary Fig. S1), these data indicate that OsNPF2.2 is a plasma membrane nitrate transporter.

OsNPF2.2 expression is induced by exogenous nitrate. Arabidopsis $N P F$ genes are expressed differently in response to nitrate supply and $\mathrm{N}$-starvation ${ }^{2}$. Transporters are also involved in the sensing of nitrate ${ }^{9}$. We therefore performed qPCR to check the transcriptional response of OsNPF2.2 to nitrate and ammonium treatment (Fig. 3a). OsNPF2.2 expression was upregulated in leaf blades in response to 1$2 \mathrm{~h}$ of $10 \mathrm{mM}$ nitrate resupply after $1 \mathrm{~d}$ nitrate deprivation (Fig. 3a). Moreover, in the roots and the leaf sheaths, OsNPF2.2 expression was slightly induced by $10 \mathrm{mM}$ nitrate resupply. In contrast, in all the tissues tested, OsNPF2.2 expression was not significantly affected by $\mathrm{NH}_{4}{ }^{+}$treatment (Fig. 3a). To further confirm that nitrate upregulated OsNPF2.2 expression in leaf blades, we examined GUS staining in pOsNPF2.2-uidA transgenic rice after $\mathrm{NO}_{3}{ }^{-}$induction (Fig. $3 \mathrm{e}-\mathrm{g}$ ). Before $\mathrm{NO}_{3}{ }^{-}$deprivation, GUS staining was present in the leaves of both $p O s N P F 2.2-u i d A$ and $p 35 S$-uidA transgenic rice plants (Fig. $3 \mathrm{~b}$, e). However, when the plants were transferred into an $\mathrm{NO}_{3}{ }^{-}-$ deprived solution for $1 \mathrm{~d}$, GUS staining disappeared from the leaves of the $p O s N P F 2.2$-uidA plants (Fig. 3f) but remained in the leaves of the $p 35 S$-uidA plants (Fig. 3c). Then, when the plants were transferred back the $\mathrm{NO}_{3}{ }^{-}$solution, the GUS staining appeared in the leaves of the $p O s N P F 2.2$-uidA plants (Fig. 3g). These results show that OsNPF2.2 expression is induced by exogenous nitrate, or repressed by nitrate starvation, different from OsNPF $2.4^{36}$ which was induced by $\mathrm{N}$ starvation in the older leaf blades.

OsNPF2.2 is mainly expressed in parenchyma cells of the vasculature. Three approaches were used to examine the expression pattern of OsNPF2.2. First, the microarray data from RiceXPro (http:// ricexpro.dna.affrc.go.jp/) showed that OsNPF2.2 is mainly expressed in leaves, roots, stems, inflorescences, anthers, pistils, lemmas, paleas and ovaries (Supplementary Fig. S2). Second, whole-mount GUS staining showed that although OsNPF2.2 is expressed in all the organs examined (Fig. 4a-f), it is preferentially expressed in the central stele in roots (Fig. 4a, b) and in leaf veins (Fig. 4e). Third, to determine the tissue-specific expression pattern of OsNPF2.2, cross- or longitudinal sectioning of the GUS-stained organs was performed (Fig. 4g-1). Sectioning confirmed that OsNPF2.2 is mainly expressed in leaf veins (Fig. $4 \mathrm{~g}, \mathrm{~h}$ ), stem vasculature (Fig. 4i, j, and k), and in the stele in roots (Fig. 4l). However, strong GUS staining was observed in vascular parenchyma cells (Fig. $4 \mathrm{~g}-\mathrm{i}, 4 \mathrm{l}$ ). Transverse and longitudinal sectioning of the stems and the leaves revealed that OsNPF2.2 is expressed in the parenchyma cells abutting xylem vessels (Fig. 4g, j, and k). Taken 


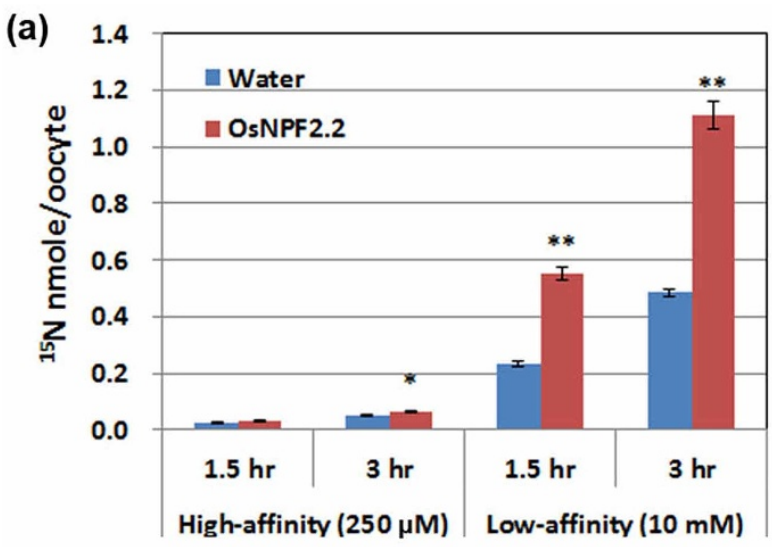

(b)

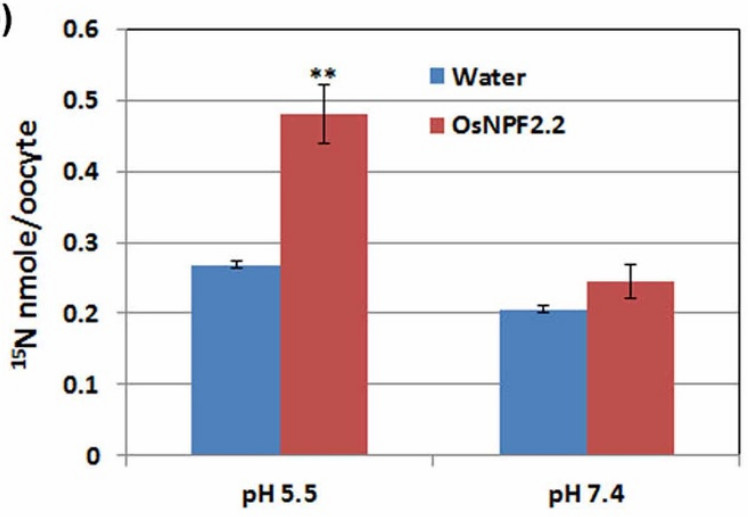

(c)

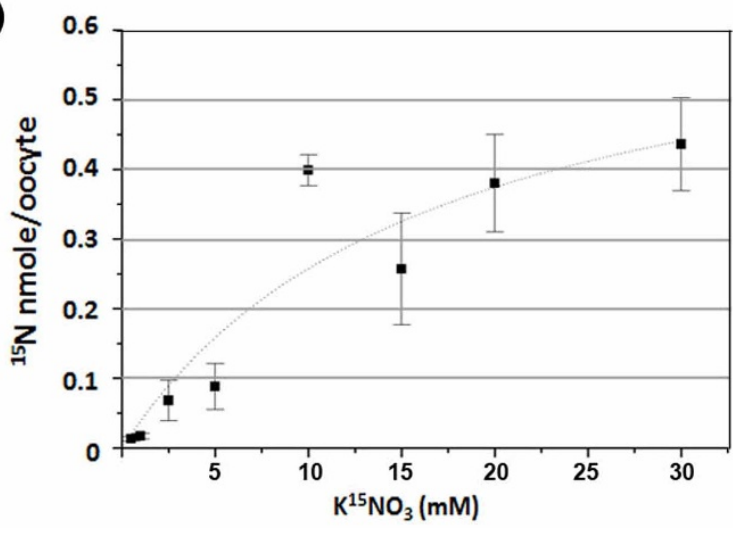

Figure $1 \mid$ OsNPF2.2 is a low-affinity nitrate transporter. (a) Nitrate uptake activity of OsNPF2.2-injected oocytes at pH 5.5. High-affinity or low-affinity nitrate uptake activity was examined by incubating oocytes with 0.25 or $10 \mathrm{mM} \mathrm{K}{ }^{15} \mathrm{NO}_{3}$ for $1.5 \mathrm{~h}$ and $3 \mathrm{~h}$ and then measuring the levels of ${ }^{15} \mathrm{~N}$ in the oocytes $(\mathrm{n}=8-13$ oocytes for the water- and OsNPF2.2-injected oocytes, respectively). (b) The $\mathrm{pH}$ dependence of the nitrate uptake activity of OsNPF2.2. The OsNPF2.2-injected oocytes were incubated with $10 \mathrm{mM} \mathrm{K}{ }^{15} \mathrm{NO}_{3}$ buffered at $\mathrm{pH} 5.5$ or 7.4 for $2 \mathrm{~h}$, and then the levels of ${ }^{15} \mathrm{~N}$ in the oocytes were measured $(\mathrm{n}=10-13$ oocytes for the water- and OsNPF2.2-injected oocytes, respectively). (c) Uptake kinetics of OsNPF2.2. OsNPF2.2-injected oocytes were

incubated with different concentrations of $\mathrm{K}^{15} \mathrm{NO}_{3}$ at $\mathrm{pH} 5.5$ for $1.5 \mathrm{~h}$, and then their ${ }^{15} \mathrm{~N}$ contents were determined. The $K_{\mathrm{m}}$, calculated from one experiment by fitting the data to the Michaelis-Menten equation using a nonlinear least squares methods in the ORIGIN 5.0 program (Microcal Software; GE Healthcare), was $\sim 16.6 \pm 12.9 \mathrm{mM}$. The values are mean $\pm \mathrm{SE}\left(\mathrm{n}=4-9\right.$ oocytes for each concentration). ${ }^{*} P<$ 0.05 when compared to the water-injected control; ${ }^{* *} P<0.01$ when compared to the water-injected control. together with the previous results, these observations show that OsNPF2.2 is strongly expressed in the parenchyma cells of vasculature in response to nitrate application.

Disruption of OsNPF2.2 hinders plant growth and seed filling. To investigate the function of OsNPF2.2, we identified two japonica rice (Oryza sativa L.) mutants, osnpf2.2-1 (3D-02162) and osnpf2.2-2 (3A-07557), which were T-DNA knockouts developed from the Hwayoung and Dongjin varieties, respectively ${ }^{41,42}$. Sequencing of the genomic fragment flanking the T-DNA insertion sites in the two mutants confirmed that the T-DNA was inserted in the OsNPF2.2 coding region in osnpf2.2-1 (3D-02162), and in the OsNPF2.2 3' - untranslated region in osnpf2.2-2 (3A-07557; Fig. 5a). T-DNA copy number analysis showed that there was a single T-DNA locus in osnpf2.2-1, and two in osnpf2.2-2 (data not shown). OsNPF2.2 transcripts were not detected in either mutant (Fig. 5b). Since most of the seeds from the two osnpf2.2 mutants were abnormal, we failed to obtain complemented lines from the mutants. However, a few homozygous mutants were identified by PCR screening and used for analysis. Homozygous osnpf2.2-1 and osnpf2.2-2 mutants had similar phenotypes in our analysis. In addition, to validate results from the two osnpf 2.2 mutants, we also generated OsNPF2.2 knockdown mutants by RNAi under the control of the rice OsNPF2.2 promoter. We found that eight independent OsNPF2.2-RNAi transgenic lines exhibited a reduction in OsNPF2.2 transcript level (Supplementary Fig. S3a).

The osnpf2.2 mutants displayed several phenotypic alterations throughout their lifespans when grown under normal $\mathrm{N}$ condition (Fig. 5c-5n). Unlike wild-type seeds, most of the seeds from the osnpf2.2 mutants did not germinate on wet tissues. When germinated on $1 / 2$ MS medium, the seed germination rates for osnpf2.2-1 and osnpf2.2-2 were obviously lower than that of their parental varieties (Fig. 5c-f). The osnpf2.2 mutant plants were dwarf (Fig. 5g, j, and m), and the panicles were short (Fig. $5 \mathrm{~h}, \mathrm{k}$ ). Grain filling in the osnpf2.2 mutants was severely blocked (Fig. $5 n$ ). The altered phenotypes of the two osnpf2.2 mutants were similar, even though the osnpf2.2-2 mutant had two copies of T-DNA. Growth retardation was also observed in the mutant plants when grown under low or high nitrate application (Supplementary Fig. S4). Most of the osnpf2.2 mutant phenotype was seen in the OsNPF2.2-RNAi transgenic plants, including dwarfism, short panicles, and low seed setting (Supplementary Fig. S3b-f). Based on the above data, we concluded that the phenotypic alterations observed in the osnpf2.2 mutants were caused by the mutations in OsNPF2.2. Therefore, we used homozygous mutant, osnpf2.2-1 and osnpf2.2-2 plants for further analysis.

Next, we carefully checked seed development in the osnpf2.2 mutants (Fig. $5 \mathrm{n}$ ). To determine whether the low rate of seed setting in the osnpf 2.2 mutants is caused by semisterile pollen or fertilized but aborted ovaries, we counted the number of the fully filled seeds, unfilled seeds with expanded ovaries (UFS), and empty spikelets per plant (Fig. 5i, 1). The data show that, for every mutant plant, the percentage of UFS is much higher than for the wild-type plant (Fig. 5n). UFS are seeds that contain fertilized egg cells but were unfilled. In addition, even the fully filled seeds of the mutants may be abnormal, since they did not germinate normally (Fig. 5c-f). Therefore, the disruption of OsNPF2.2 severely affects the development of rice seeds.

Although the two osnpf2.2 mutants and the OsNPF2.2-RNAi rice showed similar phenotypes, some differences were also observed (Fig. 5, Supplementary Fig. S3). These differences might be caused by differences in the genetic background of the two varieties and/or the different placement of the T-DNA insertion sites (Fig. 5a).

Effect of OsNPF2.2 on root-to-shoot nitrate transport. To investigate whether the osnpf2.2 mutants are defective in longdistance transport of nitrate, the nitrate concentration in the xylem 

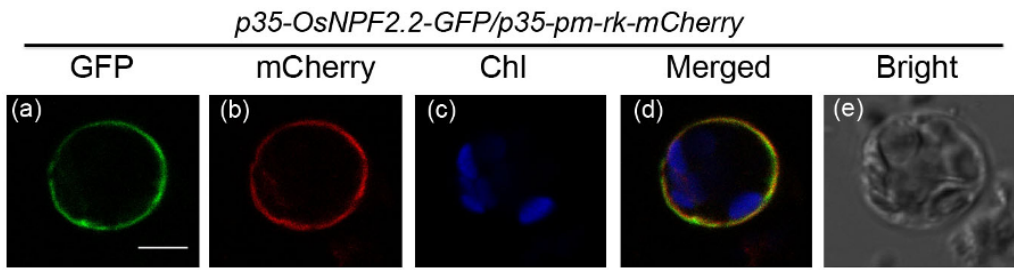

p35S-GFP

Free GFP

Figure $2 \mid$ OsNPF2.2 is located in the rice plasma membrane. (a-e) Fluorescence of OsNPF2.2-GFP coexpressed with the plasma membrane marker pmrk-mCherry in transiently transformed rice protoplasts $(\mathrm{bar}=5 \mu \mathrm{m})$. Transformed rice protoplasts were first identified by their green fluorescent protein (GFP) fluorescence from OsNPF2.2-GFP (a); then, these cells were checked for mCherry fluorescence (b) and finally for chlorophyll autofluorescence (c). The corresponding bright-field image is shown in (e). (d) Merged images from (a), (b), and (c). (f) GFP fluorescence from free GFP expressed under the $35 S$ promoter as a control (bar $=5 \mu \mathrm{m})$.

exudates, the total nitrate concentration, and the distribution of nitrate between roots and shoots were analyzed (Fig. 6). When plants were continuously grown with sufficient nitrate solution, the nitrate concentrations of the xylem sap in the osnpf2.2 mutants were significantly lower than those in the wild-type plants (Fig. 6a). In addition, more nitrate accumulated in the roots of the mutants than in the roots of the wild-type plants (Fig. 6b), and although the shoot: root nitrate ratio was 1.18 or 0.89 in the wild-type plants, it was reduced to 1.10 or 0.73 in osnpf2.2-1 and osnpf2.2-2 mutant plants, respectively (Fig. 6c). These observations show that in osnpf2.2

(a)
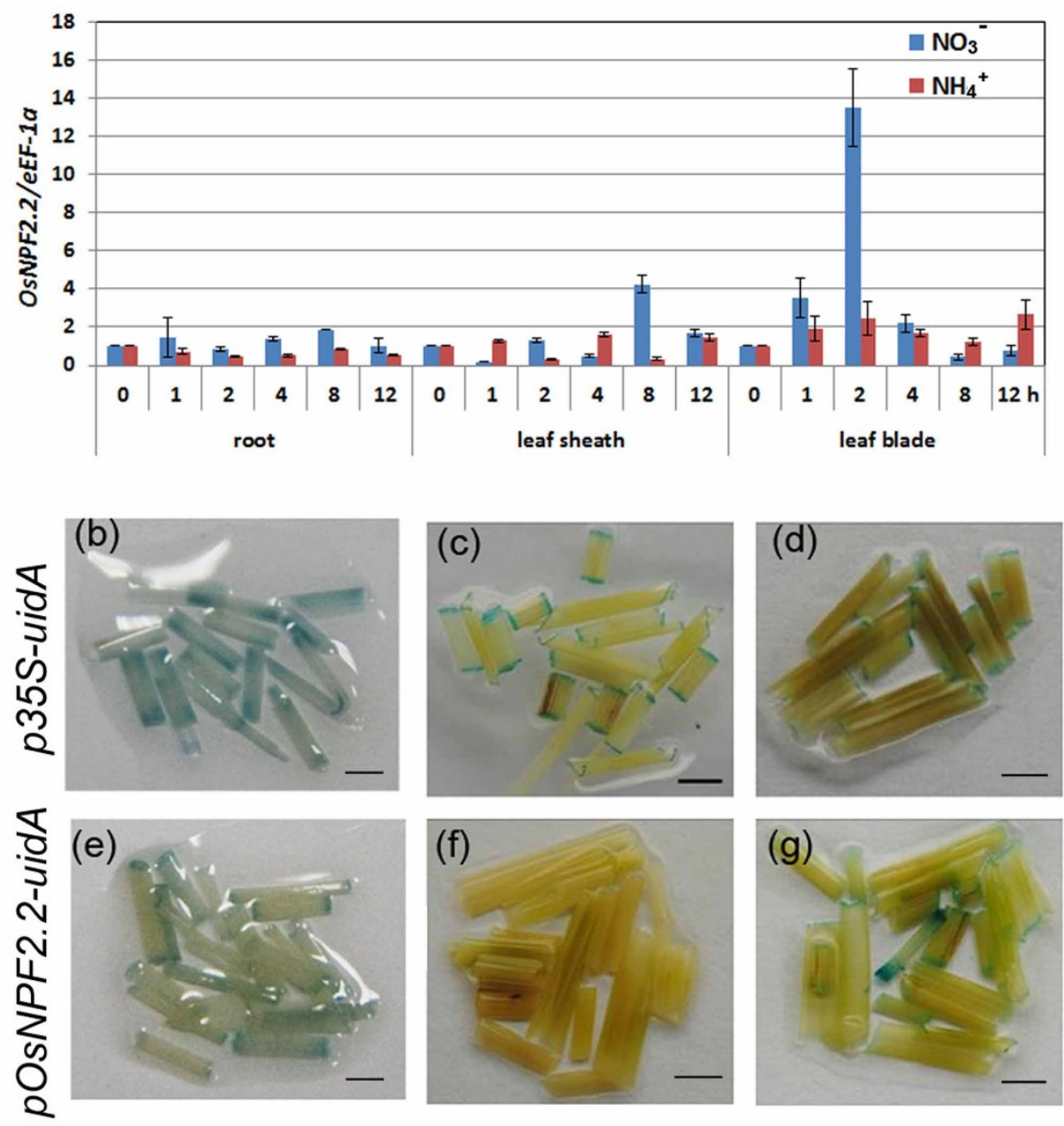

Figure 3 OsNPF2.2 expression in leaf blades is nitrate inducible. (a) Time-course analysis of $\mathrm{OsNPF}_{2.2}$ expression following $\mathrm{NO}_{3}{ }^{-}$and $\mathrm{NH}_{4}{ }^{+}$ induction by quantitative real-time-PCR. Rice seedlings were grown on 1/2 Murashige and Skoog solid medium for $10 \mathrm{~d}$; next, seedlings were washed and deprived of $\mathrm{NO}_{3}{ }^{-}$for $1 \mathrm{~d}$ and then subcultured in International Rice Research Institute (IRRI) solution with $10 \mathrm{mM} \mathrm{KNO}_{3}$ or $10 \mathrm{mM} \mathrm{NH}_{4} \mathrm{Cl}$ as the $\mathrm{N}$ source, respectively, or mock-treated with $\mathrm{KCl}$. The plants were collected for RNA extraction at the indicated times. Relative expression was normalized to the expression level of $e E F-1 a$. The values are mean \pm SE for triplicate samples, with each replicate containing seven seedlings. (b-g) $\beta-G l u c u r o n i d a s e$ activity analysis in leaf blades of the transgenic rice plants harboring the uidA gene driven by the cauliflower mosaic virus $35 \mathrm{~S}$ promoter (b- $\mathrm{d}$ ) or the $2.5 \mathrm{~kb}$ OsNPF2.2 promoter (e-g) after nitrate induction. The transgenic plants were first grown in $1.4 \mathrm{mM} \mathrm{NH}_{4} \mathrm{NO}_{3}$ solution $(\mathrm{b}, \mathrm{e})$ and then deprived of $\mathrm{NO}_{3}{ }^{-}$ for $1 \mathrm{~d}(\mathrm{c}, \mathrm{f})$; finally, the plants were transferred back into IRRI solution with $10 \mathrm{mM} \mathrm{KNO}_{3}$ as the $\mathrm{N}$ source $(\mathrm{d}, \mathrm{g})$ for $2 \mathrm{~h}$. Bar $=0.25 \mathrm{~cm}$. 
(a)

(e)

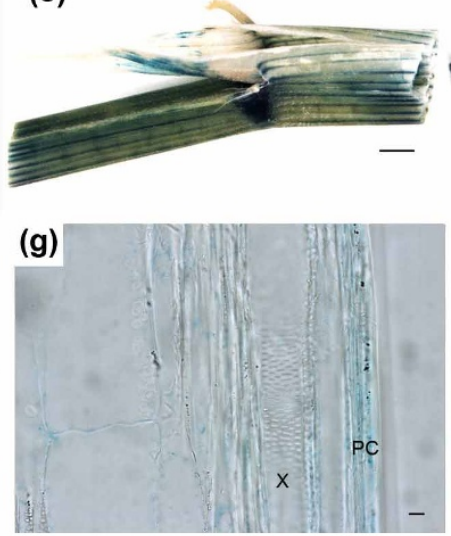

(b)

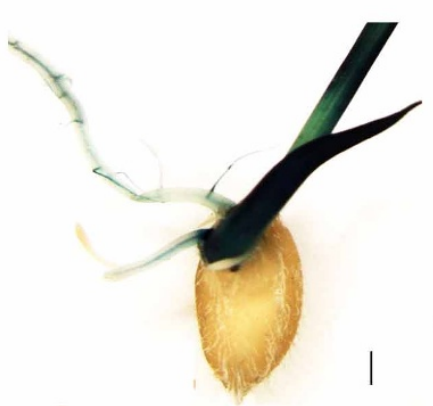

(c)

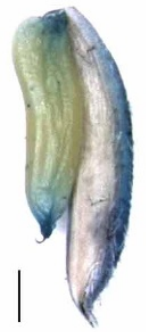

(d)

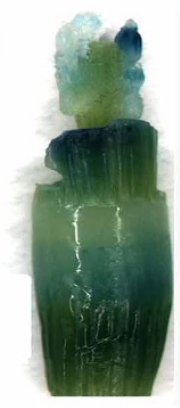

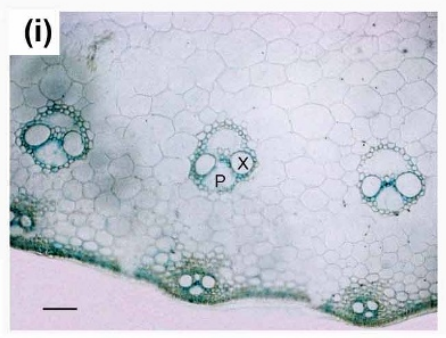

(k)

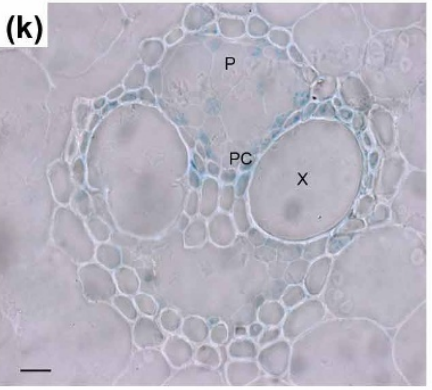

(f)

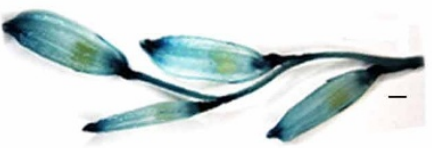

(h)
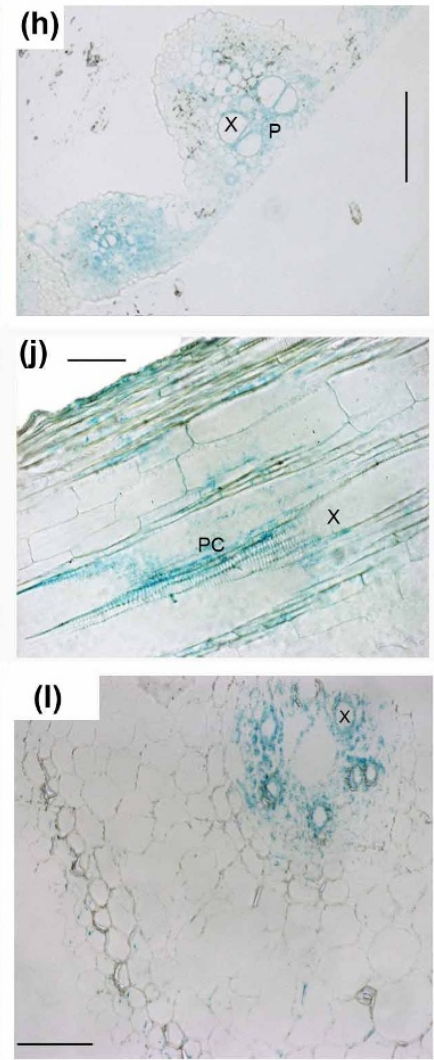

Figure $4 \mid$ OsNPF2.2 is strongly expressed in parenchyma cells around xylem vessels. (a-f) OsNPF2.2 expression patterns in various organs by $\beta$ glucuronidase staining analysis, bar $=1000 \mu \mathrm{m}$. The transgenic plants harboring the uidA gene driven by the $2.5 \mathrm{~kb} O s N P F 2.2$ promoter were grown in a paddy with normal nitrogen fertilizer. GUS activity was detectable in the roots and leaves of germinating seeds (a), in old roots (b), in filling seeds (c), in young inflorescences and stems (d), in flag leaves (e), and in panicles (f). (g-l) Longitudinal (g, j) and transverse (h, i, k, and l) section analysis of GUSstained plants transgenic for $p O s N P F 2.2-u i d A$ and grown under normal nitrate conditions. GUS staining revealed that OsNPF2.2 is strongly expressed in vascular parenchyma cells in leaves (g, h), stems (i, j, k), and roots (l). X, xylem; P, phloem; PC, parenchyma cell. Bar $=5 \mu \mathrm{m}$ in $(\mathrm{g}), 100 \mu \mathrm{m}$ in $(\mathrm{h}), 50 \mu \mathrm{m}$ in $(\mathrm{i}, \mathrm{j}$ and $\mathrm{l})$, and $10 \mu \mathrm{m}$ in $(\mathrm{k})$.

mutants, more nitrate is retained in the roots and less nitrate is transported into the shoots than in wild-type plants. Taken together, these results suggest that OsNPF2.2 plays a role in transporting nitrate from the roots to the shoots.

Disruption of OsNPF2.2 affects unloading nitrate from xylem. Since OsNPF2.2 is expressed in the parenchyma cells abutting the xylem, it may be involved in nitrate loading into the xylem, like AtNPF7. $3^{19}$, or nitrate unloading from the xylem, like AtNPF7.20 therefore, disruption of OsNPF2.2 should affect the accumulation of nitrate in the xylem sap. To test this hypothesis, the xylem exudates of plants were collected and analyzed (Fig. 7a) after a $2 \mathrm{~h}$ nitrate feeding following $\mathrm{NO}_{3}{ }^{-}$-deprivation, as follows. Plants grown in rich nitrate solution were transferred to $\mathrm{NO}_{3}{ }^{-}$-depleted solution (with $\mathrm{NH}_{4} \mathrm{SO}_{4}$ as the $\mathrm{N}$ source) for 1 week to remove internal nitrate. Then, the $\mathrm{NO}_{3}{ }^{-}$-starved plants were transferred into $10 \mathrm{mM}$ nitrate IRRI solution for $2 \mathrm{~h}$, after which the xylem exudates from shoots with roots were collected. The nitrate concentration in xylem sap was greater in the osnpf2.2 mutants than in the wild-type plants (Fig. 7a). These data indicate that under short-term nitrate resupply after $\mathrm{NO}_{3}{ }^{-}$-starvation, more nitrates is accumulated in the xylem vessels of the osnpf2.2 mutant than in those of the wildtype plant, suggesting that OsNPF2.2 may also play a role in unloading nitrate from the xylem. Next, the root and shoot nitrate contents of plants that had been fed with nitrate for $2 \mathrm{~h}$ were measured. The nitrate concentrations in the roots and the shoots of the osnpf2.2 mutants were greater than those in the wild-type plant (Fig. 7b); this observation indicated that more nitrate was retained in roots and shoots in the osnpf 2.2 mutants than in the wild-type plant. The reason for this result may be that less nitrate is unloaded from the xylem for assimilation in the roots and the shoots under short-term nitrate feeding after $\mathrm{NO}_{3}{ }^{-}$-starvation in the mutant plants than in the wild-type plant. Taken together with the observation of $p O s N P F 2.2-G U S$ staining in the parenchyma cells of the vasculature (Fig. 4), these results suggest that disruption of OsNPF2.2 may block nitrate unloading from the xylem.

The osnpf2.2 mutants display abnormal vasculature. OsNPF2.2 is highly expressed in the vasculature of all organs (Fig. 4), and the xylem and the phloem in the vasculature are important for nitrate 

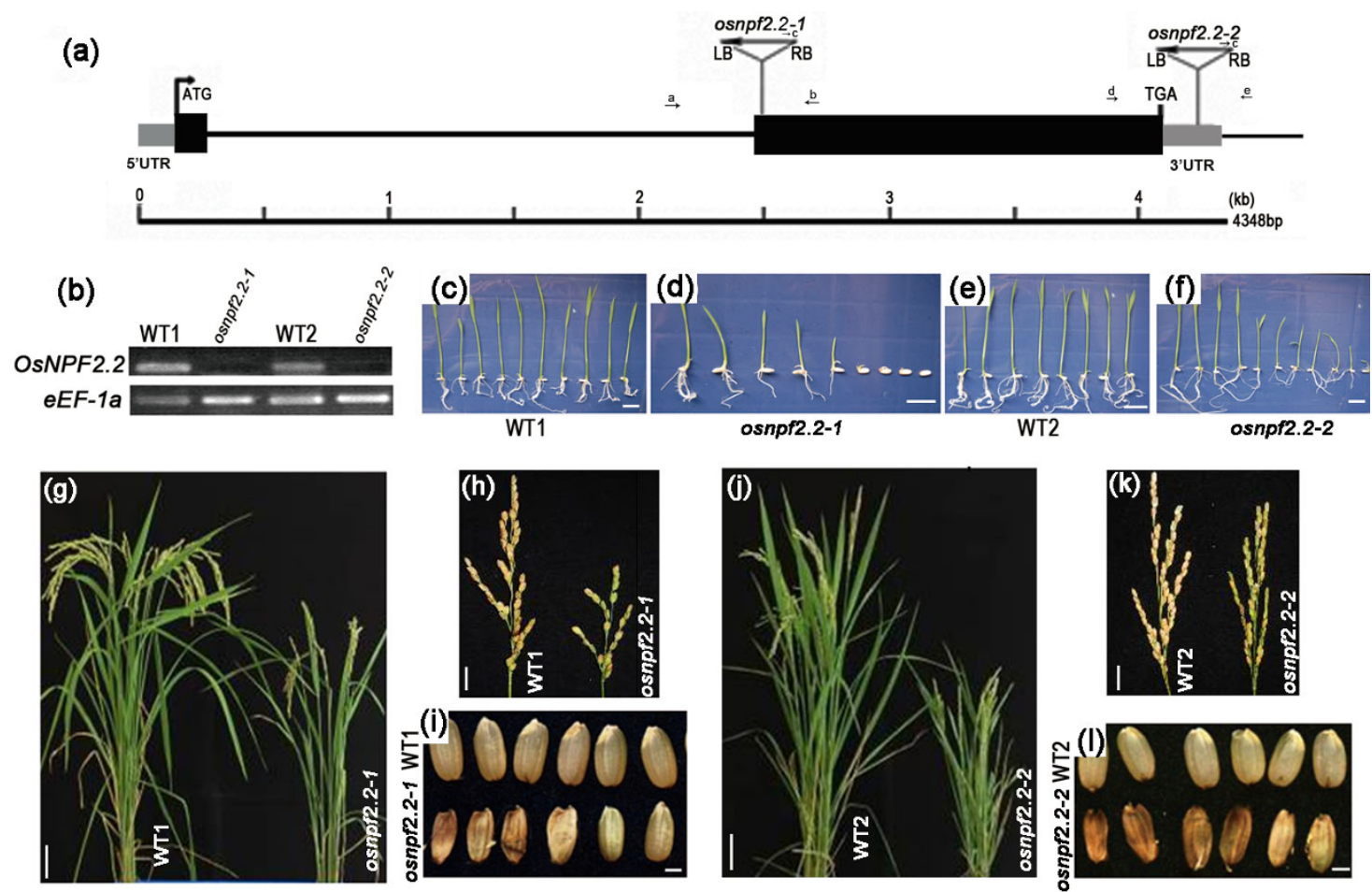

$(\mathrm{m})$
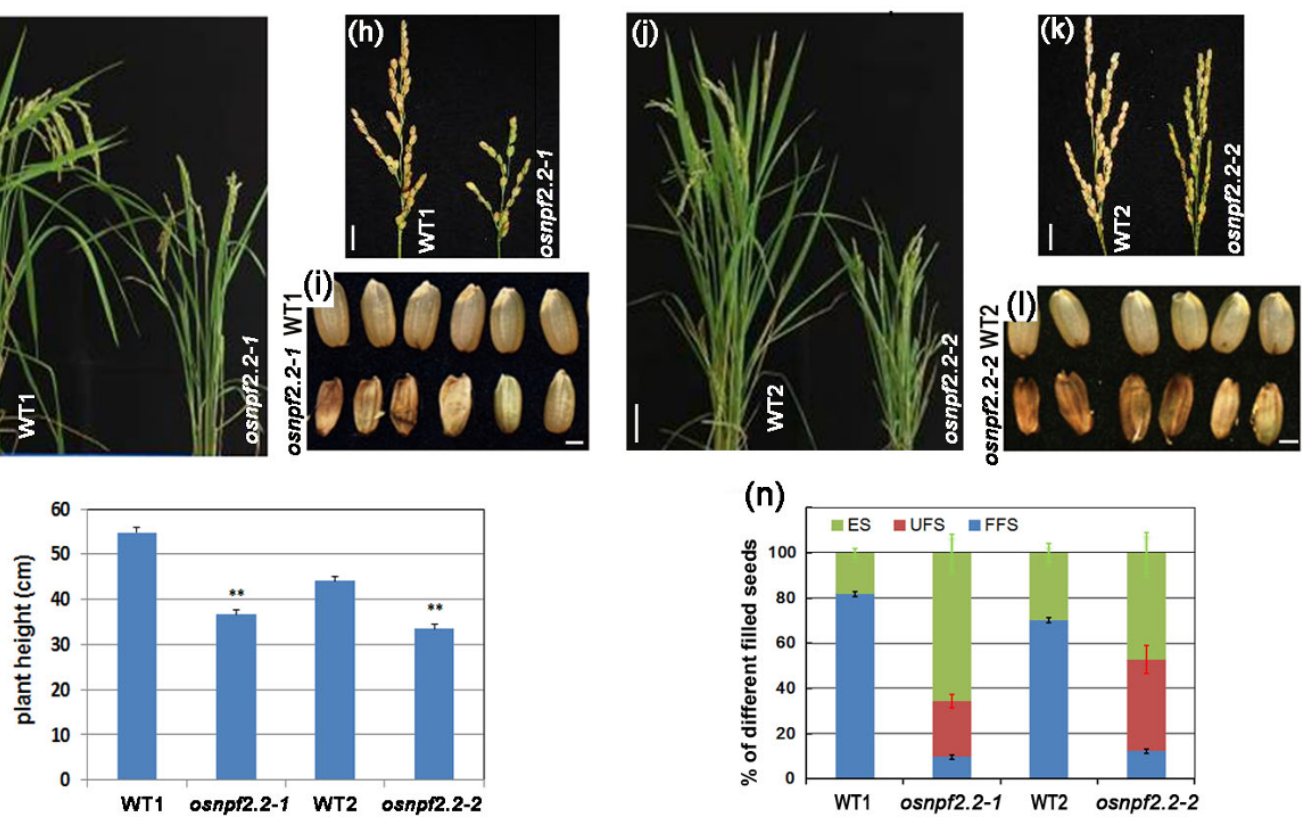

Figure 5 Disruption of OsNPF2.2 hinders plant growth and seed filling. (a) Schematic diagram of OsNPF2.2 and insertion positions of transfer DNA (T-DNA) in the osnpf2.2-1 and osnpf2.2-2 mutants. The boxes indicate two exons, and the connecting black line represents the intron. Black boxes indicate the open reading frame, and the gray boxes indicate the $5^{\prime}$ - and $3^{\prime}$ - untranslated regions. The T-DNA is indicated by the triangle. The abbreviations LB and RB indicate the left and right borders, respectively, of the T-DNA. Arrows with letters show the locations of the primers used to amplify the flanking sequence tags and screen the homozygous mutants. (b) Semi-quantitative-PCR analyses of OsNPF2.2 expression in osnpf2.2 mutants. Total RNA was extracted from the leaves of homozygous osnpf2.2-1 and osnpf2.2-2 plants and their parental (WT) varieties. The gene $e E F-1 a$ was amplified as an internal control. (c-f) Seed germinating comparison between the osnpf2.2 mutants and WT plants. The surface-sterilized seeds were germinated on 1/2 Murashige and Skoog solid medium; bar $=2 \mathrm{~cm}$. (g-n) Main phenotypes of the osnpf2.2 mutants grown in a paddy with normal nitrogen fertilizer. (g, j) Dwarf plants; bar $=5 \mathrm{~cm} ;(\mathrm{h}, \mathrm{k})$ Short panicles; bar $=1.5 \mathrm{~cm} ;(\mathrm{i}, \mathrm{l})$ Reduction in filling for seeds from the osnpf2.2 mutants as compared to WT seeds; bar $=$ $2 \mathrm{~mm}$. (m) Plant height statistics of the osnpf2.2 mutants and WT plants at the mature stage; ${ }^{* *} P<0.01$ when compared to WT plants. (n) Comparison analysis of the fully filled seeds (FFS), the unfilled seeds with expanded ovaries (UFS), and the empty spikelets (ES) per plant between the osnpf2.2 mutants and WT plants. The values are mean \pm SE from 60 plants at two seasons.

transport ${ }^{2}$. To determine whether mutations in OsNPF2.2 affect vascular development, we examined the vasculature in osnpf2.2 mutants by sectioning. All the tested organs displayed abnormal vascular bundles (Supplementary Fig. S5). For example the external vascular bundles in the stems of the osnpf2.2 mutants displayed abnormal structure (Supplementary Fig. S5b, d) as compared to those in wild-type plants, in which stems contain rings of regular external vasculatures (Supplementary Fig. S5a, c). The anthers and branches in osnpf2.2-1 mutant plants also had disturbed sieve tubes and vessels (Fig. 8b, f). When examining the ultrastructure of the osnpf2.2 mutants, we found that there was still some cytoplasm in a few vessel cells in the osnpf2.2-1 leaves (Fig. 8d); this observation indicated that programmed cell death in some xylem vessels was blocked. From the sections, we concluded that disruption of OsNPF2.2 disturbs vasculature development and thus affects the long-distance transport of nitrate and other metabolites.

\section{Discussion}

The sustainable production of food to feed the world's population is critical for both human and environmental health, and the study of plant membrane transporters may be useful for enhancing the yields of staple crops ${ }^{43}$. In the large NPF transporter family, a few members have been functionally characterized as transporters for nitrate, peptides, glucosinolates, indole-3-acetic acid, and abscisic acid ${ }^{10,18}$; however, most of the data about these transporters has been obtained from studies in Arabidopsis. Rice is one of the most important cereals ${ }^{30}$, and it expresses 93 NPF family members ${ }^{10}$. However, only OsNPF8.9 and OsNPF2.4 is known to transport nitrate ${ }^{35,36}$ and physiological role of OsNPF8.9 in rice is still unknown. We showed here that OsNPF2.2 is a low-affinity nitrate transporter by expressing OsNPF2.2 in Xenopus laevis oocytes, and by showing that a loss-offunction mutation for OsNPF2.2 resulted in the accumulation of nitrate in the xylem, the reduction of nitrate transport from roots to shoots, and retardation of rice growth. Taken together with the 
(a)

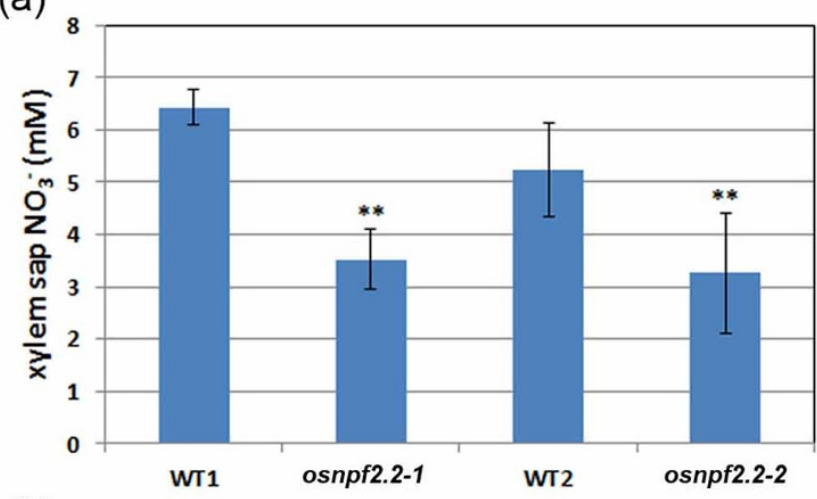

(b)

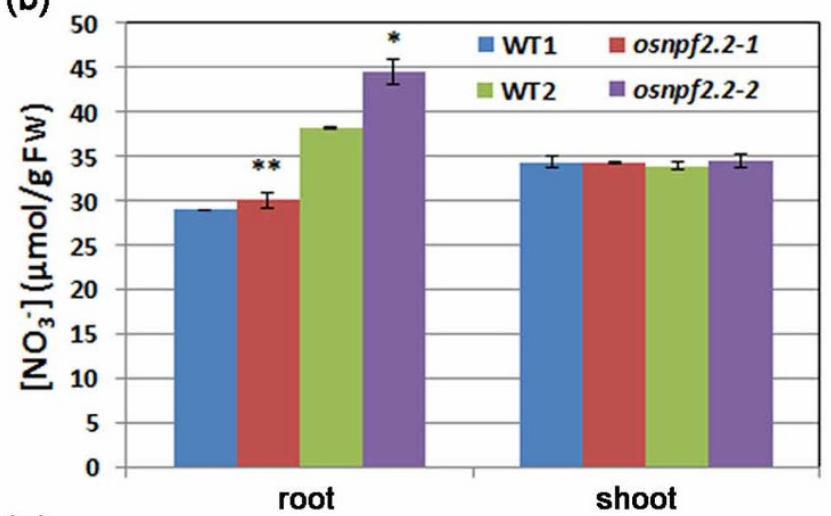

(c)

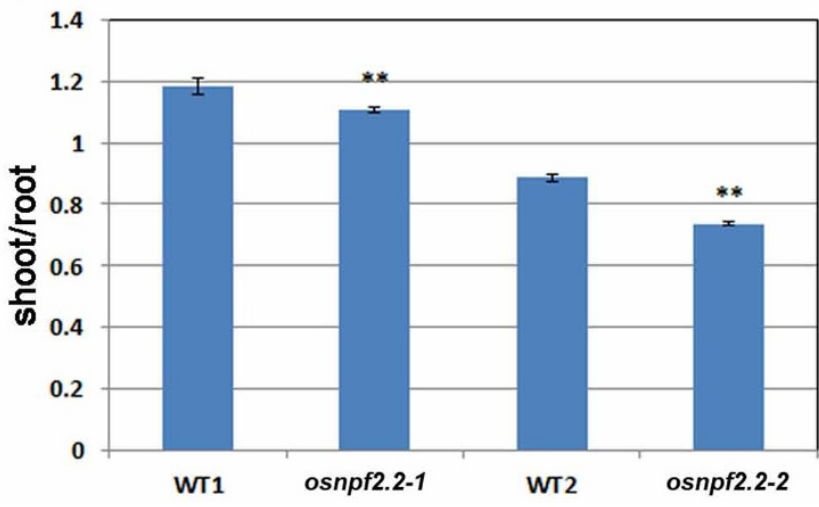

Figure 6 | Disruption of OsNPF2.2 affects nitrate transport from root to shoot. $(a-c)$ Nitrate concentrations in the xylem exudate (a), total nitrate concentration in the roots and shoots (b), and the shoot:root nitrate ratio (c) of the wild-type (WT) plants and the osnpf2.2 mutants. The plants were grown in International Rice Research Institute (IRRI) solution containing $1.4 \mathrm{mM} \mathrm{NH}_{4} \mathrm{NO}_{3}$ for 8 weeks for the steady-state nitrate treatment. To collect the xylem sap, the plants were cut $4 \mathrm{~cm}$ above the roots, and the roots were immediately transferred to $10 \mathrm{mM}$ nitrate IRRI solution for $2 \mathrm{~h}$. Xylem sap was collected over the $2 \mathrm{~h}$ period. To measure the total nitrate concentration in the roots and shoots, the samples were directly harvested after cultivation. The values represent mean \pm SE for triplicate samples; ${ }^{*} P<0.05$ when compared to WT plants; ${ }^{*} P<0.01$ when compared to WT plants.

observation that OsNPF2.2 is expressed in parenchyma cells around xylem vessels, the data indicates that OsNPF2.2 unloads nitrate from the xylem to transport nitrate from the roots to shoots.

The rice genome contains 93 NPF members, which is more than that of Arabidopsis ${ }^{10}$. In our prior study, we found that OsNPF2.2 expression is nitrate inducible and that OsNPF2.2 could not transport peptides ${ }^{44}$; mutation of OsNPF2.2 caused serious abnormal

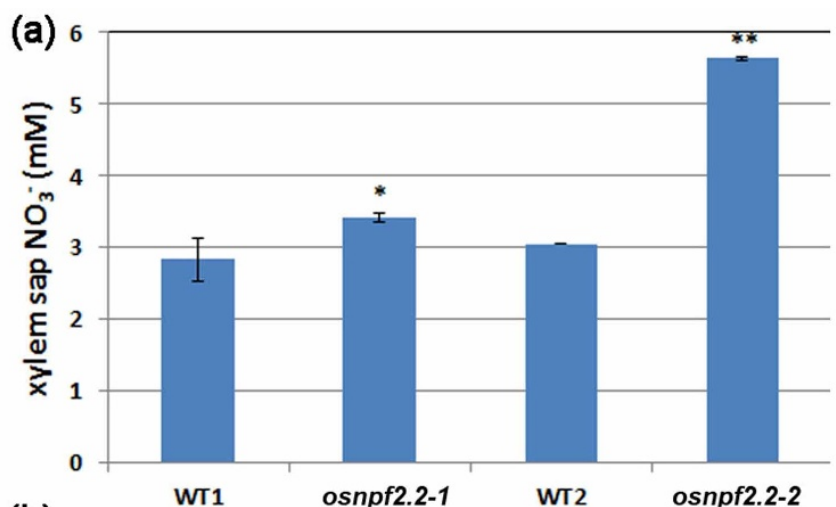

(b)

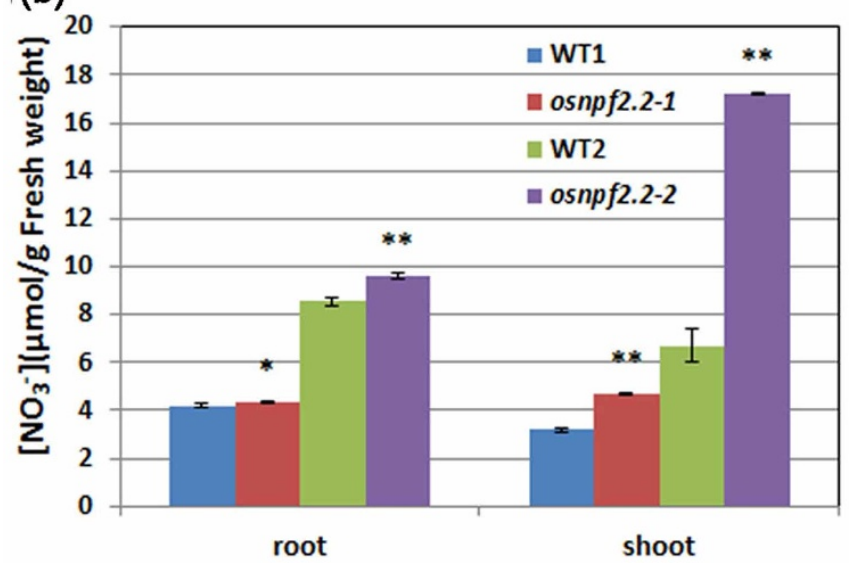

Figure 7 Disruption of OsNPF2.2 affects nitrate unloading from the xylem. (a, b) Nitrate concentrations in the xylem exudates (a), and total nitrate concentration in the roots and shoots (b) of wild-type (WT) plants and the osnpf2.2 mutants under $2 \mathrm{~h}$ short-term nitrate feeding. The plants were grown in International Rice Research Institute (IRRI) solution containing $1.4 \mathrm{mM} \mathrm{NH}_{4} \mathrm{NO}_{3}$ for 8 weeks; next, they were transferred to IRRI solution containing $1.4 \mathrm{mM} \mathrm{NH}_{4} \mathrm{SO}_{4}$ for $\mathrm{NO}_{3}$-starvation for 1 week and then transferred back to IRRI solution with $10 \mathrm{mM} \mathrm{KNO}_{3}$ for $2 \mathrm{~h}$ for xylem sap collection (a) or for measuring the total nitrate concentration in the roots and shoots (b). The values are mean \pm SE for triplicate samples; ${ }^{*} P<0.05$ as compared to WT plants; ${ }^{* *} P<0.01$ as compared to WT plants.

growth. These data indicated that OsNPF2.2 might be important for rice nitrate transport. Therefore, in this study, OsNPF2.2 was analyzed by expression in Xenopus laevis oocytes to test its nitrate transport capacity. In Xenopus laevis oocytes, OsNPF2.2 showed uptake ability for nitrate at high nitrate levels, and this nitrate uptake activity was $\mathrm{pH}$ dependent (Fig. 1). The various Arabidopsis NPF nitrate transporters function as influx, efflux, or bidirectional nitrate transporters ${ }^{18}$. Our data show that OsNPF2.2 has nitrate influx capacity, but we do not know whether it also has nitrate efflux capacity. OsNPF2.2 is clustered in the NPF2 subfamily of the NPF family ${ }^{10}$. This clade also contains AtNPF2.10 and AtNPF2.11, which are glucosinolate transporters ${ }^{11}$. Therefore, it will be important to further analyze whether rice contains glucosinolates, and whether OsNPF2.2 can transport glucosinolates.

Transporters for nitrate loading into xylem vessels and unloading from xylem vessels were previously identified in Arabidopsis ${ }^{2,19,20}$. In the present study, we found that OsNPF2.2 is strongly expressed in the parenchyma cells of the vasculature (Fig. 4) and that disruption of OsNPF2.2 led to nitrate accumulation in the xylem sap of osnpf2.2 mutants (Fig. 7a) when nitrate is resupplied after $\mathrm{NO}_{3}{ }^{-}$-starvation. When nitrate was resupplied after $\mathrm{N}$-starvation, the nitrate concentration in the roots and the shoots of the wild-type plants remained at a relatively low level $(<10 \mu \mathrm{M}$; Fig. $7 \mathrm{~b})$ as compared with the rela- 

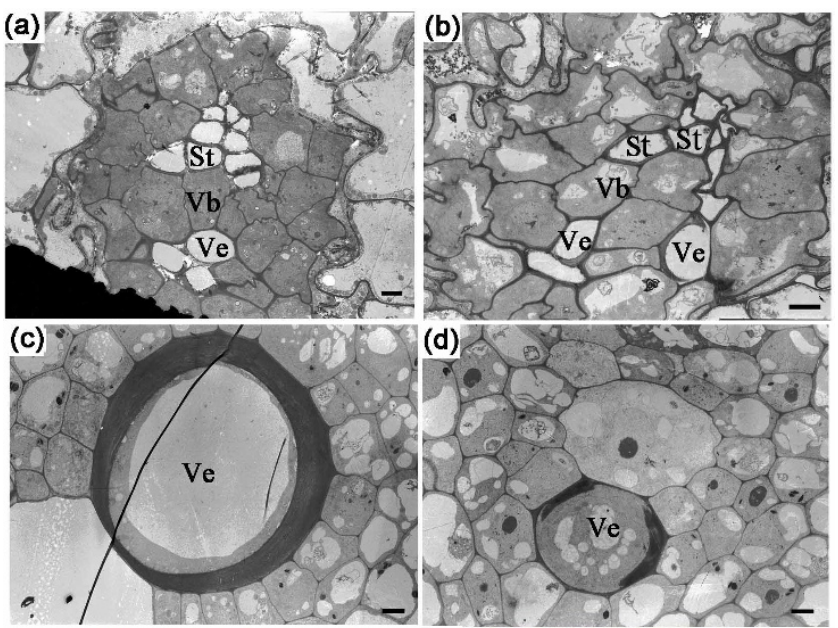

(e)
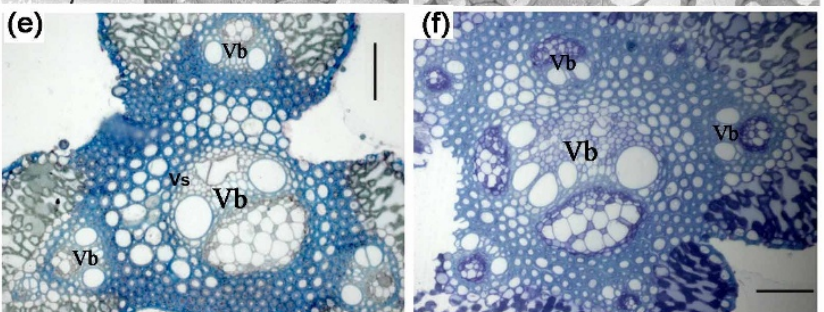

Figure $8 \mid$ Disturbed vasculatures in the osnpf2.2-1 mutants, as shown by transmission electron microscopy and semisection analysis. (a, b) Anther cross section from a wild-type (WT) plant (a) and an osnpf2.2-1 mutant (b); the sieve tubes ( $\mathrm{St}$ ) and vessels (Ve) in the osnpf2.2-1 mutant are disrupted; bar $=2 \mu \mathrm{m}$. (c, d) Ultrastructure of leaf blades from a WT plant (c) and an osnpf2.2-1 mutant (d); a few vessels in the venation in the mutant plant showed delayed dying as compared with those in the WT plant; bar $=5 \mu \mathrm{m}$. (e, f) Semisection of primary branches from a WT plant (e) and an osnpf2.2-1 mutant (f), bar $=50 \mu \mathrm{m}$. There was no obvious vascular sheath around the vascular bundle $(\mathrm{Vb})$ in the osnpf2.2-1 mutant, and its sieve tubes and vessels were irregularly distributed in the vascular bundles.

tively high level of ( $>25 \mu \mathrm{M}$; Fig. $6 \mathrm{~b})$ seen under the steady rich nitrate condition. The reason for this observation may be as follows: when nitrate was resupplied after $\mathrm{NO}_{3}{ }^{-}$-starvation, the relative low nitrate level inside the wild-type plant may have caused most of the nitrate to be unloaded from the xylem vessels and be assimilated in the roots, so that less nitrate was transported to the shoot xylem. Thus, the nitrate level in the shoot xylem exudate would be low (Fig. 7a). In contrast, in osnpf2.2 mutants under the same conditions, nitrate would have been insufficiently unloaded from the xylem and thus nitrate would have accumulated in the xylem sap, roots, and shoots of the osnpf2.2 mutants (Fig. 7). Together with the nitrate transport activity of OsNPF2.2, these observations indicate that OsNPF2.2 is responsible for unloading nitrate from the xylem to the parenchyma cells.

However, when nitrate was resupplied after $\mathrm{NO}_{3}{ }^{-}$-starvation or under steady rich nitrate conditions, the changes in xylem sap nitrate concentrations of the osnpf2.2 mutants were opposite to those seen in the wild-type plants (Fig. 6a vs. 7a). Therefore, which data could be used to explain the xylem loading and unloading? When plants are grown in low external nitrate concentration, most of the nitrate assimilation occurs in the root, whereas, under high external nitrate concentrations, shoot nitrate assimilation becomes increasingly important ${ }^{45}$. However, to determine the effect of OsNPF2.2 on the distribution of nitrate among organs, its function should be further investigated under the steady rich nitrate condition.

Plants adjust their development in relation to the availability of nutrient sources ${ }^{46}$. Although OsNPF2.2 is mainly expressed in the vasculature (Fig. 4), the disruption of OsNPF2.2 led to high nitrate concentrations in the roots of the osnpf2.2 mutants (Fig. 6b) under normal steady rich nitrate conditions. However, the shoot:root ratio (Fig. 6c) of nitrate decreased in the osnpf2.2 mutants when the plants were grown continuously under normal nitrate conditions. This observation suggests that OsNPF2.2 regulates the nitrate allocation from roots to shoots. The inhibition of nitrate transport into the leaf blades and the developing seeds in the osnpf2.2 mutants might explain their slow growth rate and the failure of seed filling (Fig. 5 and Supplementary Fig. S4). Nitrate uptake and transfer inside plants is regulated not only by external nitrate but also by intrinsic factors ${ }^{2}$. High nitrate concentrations inside the osnpf2.2 mutant plants may repress the nitrate uptake pathway via negative feedback, as occurs in the regulation of proteins such as OsNPF8.9 ${ }^{35}$, and OsNAR2. ${ }^{47}$, etc. Under nutrient limitation, an immediate response is upregulating specific transporters for nutrient uptake $\mathrm{e}^{48,49}$. OsNPF2.2 expression is induced by nitrate (Fig. 3), and it is also involved in the response to nitrate in rice. The osnpf2.2 mutants had more serious abnormal phenotypes (Fig. 5) than the sp1 mutant, for which the main phenotype was short panicles ${ }^{50}$. The OsNPF2.2 knockout mutants and the OsNPF2.2-RNAi transgenic plants had similar phenotypes (Fig. 5 and Supplementary Fig. S3). These results indicate that OsNPF2.2 is important for nitrate allocation within rice plants, and for rice normal growth.

OsNPF2.2 is also involved in the development of vascular bundles (Fig. 8 and Supplementary Fig. S5). The vascular system of the plant is a network of cells that interconnects all major plant organs. Vascular tissue formation in plants is a process with broad developmental and physiological consequences. Arabidopsis AtNPF6.3 may be a nitrate sensor that regulates root development ${ }^{13}$. Disruption of OsNPF8.20 (OsPTR9) caused the loss of some external stem vasculatures ${ }^{51}$. However, the abnormal vascular bundles in osnpf2.2 mutants are different from those in the osnpf8.20 mutant, as the vascular bundles in the osnpf 2.2 mutants (Fig. 8 and Supplementary Fig. S5) were much more seriously disrupted than those in the osnpf8.20 mutant ${ }^{51}$. In the stems of the osnpf2.2 mutants, all the external vasculature had an abnormal structure (Supplementary Fig. S5). The abnormal vascular bundles in the osnpf2.2 mutants appeared in all organs, including roots, sheaths, leaves, stems, panicle branches, and anthers (Supplementary Fig. S5). In particular, some xylem cells were not correctly broken up in the osnpf2.2 mutants (Fig. 8d). Taken together, these results indicate that OsNPF2.2 also affects the normal development of the vascular bundles. The abnormal vascular bundles in osnpf 2.2 mutants might also hinder the transport of nitrate and other metabolites. Of course, the abnormal vascular bundles might themselves be caused by the high nitrate accumulation in the osnpf 2.2 mutants.

In Arabidopsis, several low-affinity nitrate transporters (AtNPF6.3AtNPF2.9) have been shown to play various roles in nitrate transport in different parts of the plant ${ }^{2}$. Unlike OsNPF8.9, which is constitutively expressed in the most external layer of the roots, that is, the epidermis and root hairs ${ }^{35,36}$, OsNPF2.2 is constitutively expressed in most organs, even in the stele of old roots (Fig. 4), and it is induced by nitrate (Fig. 3). In addition, individual mutation of OsNPF2.4 $4^{36}$ and OsNPF2.2 (Fig. 5) could cause obvious phenotype. These suggest that the roles of OsNPF8.9, OsNPF2.4 and OsNPF2.2 are different in rice, while the three OsNPFs could transport nitrate. OsNPF2.2 is highly expressed in leaves and branches (Fig. 4 and Supplementary Fig. S1); this observation suggests that OsNPF2.2 regulates nitrate transport into leaf blades and the reproductive organs.

Nitrate can be accumulated in seeds and thus can affect dormancy in seeds ${ }^{52}$, including rice seeds ${ }^{53}$. In Arabidopsis, AtNPF2.12 $2^{24}$ and NRT2. $7^{54}$ mediate nitrate movement into seeds. OsNPF2.2 is highly expressed in reproductive organs and the young inflorescence (Fig. 4), and osnpf2.2 mutants have a high percentage of unfilled seeds (Fig. 5 and Supplementary Fig. S3). These results indicate that 
OsNPF2.2 has an important role in controlling nitrate movement into the spikelet and affects the development of the reproductive organs. The primary and secondary branches are main routes for nitrate transport into the spikelet, and OsNPF2.2 is highly expressed in the panicle branches (Fig. 4). In addition, the osnpf2.2 mutants had abnormal branches with disturbed vasculatures (Fig. 8 and Supplementary Fig. S5). These results suggest that OsNPF2.2 may control nitrate transport into the spikelet.

\section{Methods}

Plant materials, growth conditions. The japonica rice (Oryza sativa L.) mutants, osnpf2.2-1 (3D-02162) and osnpf2.2-2 (3A-07557), were transfer DNA (T-DNA) knockouts developed from the Hwayoung and Dongiin varieties, respectively, by the Korean Plant Functional Genomics Laboratory ${ }^{41,42}$. The other transgenic rice cultivar used in this study is the japonica rice variety Zhonghua 11. For hydroponic growth, plants were grown in a $20 \mathrm{~L}$ hydroponic box with an International Rice Research Institute (IRRI) rice nutrient solution ${ }^{55}$ in a growth rooms $\left(28 \pm 2^{\circ} \mathrm{C}, 14 \mathrm{~h}\right.$ light, $10 \mathrm{~h}$ dark). For soil-grown experiments, plants were grown in a rice paddy of the South China Botanical Garden, Chinese Academy of Sciences, Guangzhou, China (SCBG).

Functional analysis of OsNPF2.2 in Xenopus laevis oocytes. The $1.8 \mathrm{~kb}$ OsNPF2.2 cDNA was cloned into the oocyte expression vector pGEMHE and linearized with NheI. Capped mRNA was transcribed in vitro using mMESSAGE mMACHINE kits (Amion). Oocytes were isolated and injected with 50-100 ng of OsNPF2.2 cRNA in $50 \mathrm{~nL}$ of water; other processes were as described previously ${ }^{21}$.

Construction of vectors and plant transformation. For the OsNPF2.2 promoter- $\beta$ glucuronidase construct, the $2.5 \mathrm{~kb}$ promoter of the OsNPF2.2 gene was amplified by PCR. The following primers were used for the amplification: $5^{\prime}$

CCCAAGCTTATGAACAGTTGAGAAGAC- $3^{\prime}$ (forward) and 5 '

CATGCCATGGATTTATGTAAGATTAGGC- 3 ' (reverse). The resulting PCR fragment was inserted in the HindIII/NocI sites at the $5^{\prime}$ end of the uidA reporter gene in pCAMBIA1301 (http://www.cambia.org).

To generate the hairpin RNAi construct, a highly specific $114 \mathrm{bp}$ fragment of OsNPF2.2 was amplified by PCR using the forward primer O25F (5'GGTACCACTAGTCTAATCTTACATAAAT- $\left.3^{\prime}\right)$, and the reverse primer O25R (5' GGATCCGAGCTCGAGATGTGCTGCTGCTTC- $3^{\prime}$ ). The resulting PCR fragment was cloned in the sense orientation into the SpeI/SacI sites of pTCK $303^{56}$ to generate pTCK-114i and then, cloned in the antisense orientation into the KpnI/BamHI sites of pTCK-114i to obtain the OsNPF2.2-RNAi construct. Finally, the OsPTR2 promoter was inserted into the HindIII/BamHI sites of the OsNPF2.2-RNAi construct, replacing the UBI-1 promoter, to obtain the final vector (pTCK-pOsNPF2.2-114bpOsNPF2.2 Sense-Intron-114bp-OsNPF2.2 Antisense). The construct was transformed into Agrobacterium tumefaciens (strain EHA105) and used to transform calli developed from Zhonghua 11 seeds to obtain the transgenic rice, as previously described $^{51}$.

To generate a green fluorescent protein (GFP)-tagged OsNPF2.2 fusion protein construct ( $p 35 S-O s N P F 2.2-G F P$ ) for transient expression, OsNPF2.2 cDNA was amplified by PCR using the forward primer 5' GGATCCGCCTAATCTTACATAAAT- $3^{\prime}$ and the reverse primer $5^{\prime}$ GGATCCGACCGCCATGGCCGGCGG- ${ }^{\prime}$. The amplified fragment was then subcloned in front of the GFP coding region in the vector pUC18-GFP.

Semi-quantitative RT-PCR and quantitative real-time PCR. Total RNA was extracted using TRIzol reagent (TaKaRa, China). The first strand cDNAs were synthesized using oligo (dT) primers and MML reverse transcriptase (Promega. China). Specific semi-quantitative RT-PCR primers used to amplify OsNPF2.2 and $e E F-1 a$ were as follows: OsNPF2.2 forward, 5'-GCCGTCGCAGGAGCAAACT-3'; OsNPF2.2 reverse, 5' -CGAGGAGGAGGCACAAGGTG-3'; $e E F-1 a$

forward, $5^{\prime}$-AGCCGCTGAGATGAACAA-3'; and $e E F-1 a$ reverse, $5^{\prime}$ GAGATGGGAACGAAGGGA-3 '. Quantitative real-time PCR (qPCR) was performed using SYBR Green Premix (TaKaRa) and monitored with the 7500 RTqPCR system (Applied Biosystems, USA). The primers used for qPCR were as follows: OsNPF2.2 forward, 5' -GCAGGAGCAAACTAAGCT-3'; OsNPF2.2 reverse, 5' TGGGGATGTGGAAGACGGT-3', eEF-1a forward, 5' -GCACGCTCTTCTTGCTTTC-3'; and eEF-1 $a$ reverse, $5^{\prime}$-AGGGAATCTTGTCAGGGTTG - ${ }^{\prime}$.

Identification of the T-DNA-generated mutants osnpf2.2-1 and osnpf2.2-2. Flanking sequence tags (FSTs) for osnpf2.2-1 and osnpf2.2-2 were amplified by PCR, and the resulting products were sequenced to confirm the presence of the T-DNA in OsNPF2.2. The following primers were used to screen the mutant lines: $\mathrm{a}, 5^{\prime}-$

TGGACTGTGCCACGTGTAAT-3'; b, 5'-CCAGTTGATGTTGCTCTGGA-3'; c, $5^{\prime}$-TTGGGGTTTCTACAGGACGTAAC-3'; d, 5' -ACGGTGTTCCAGGTGATG$3^{\prime}$; and e, 5'-CAGGGTGAAGGTTTAGCAAT- $3^{\prime}$. Homozygous lines were screened by PCR using a combination of primers a, b, and c for osnpf 2.2-1 or a combination of primers d, c, and e for osnpf2.2-2. Gene expression was confirmed by RT-PCR as described above. Southern blotting was used to examine the T-DNA copy number in the two mutants. Their genomic DNAs were digested with XbaI, BamHI and HindIII and hybridized with a digoxigenin-DNA-labeled hygromycin-resistance gene probe.
Phenotypic analysis in a paddy field. Rice seeds were surfaced sterilized and germinated in 1/2 Murashige and Skoog media for 10 days; then the seedlings were transferred into an SCBG paddy field for 30 days. Finally, the seedlings were transplanted in the paddy field under normal rice growth conditions and fertilized as for normal rice growth

GUS staining for plants grown under normal $\mathrm{N}$ conditions or with nitrate induction. Homozygous $\mathrm{T}_{3}$ transgenic plants with $p O s N P F 2.2-u i d A$ or with $p 35 S$ uidA were used for GUS staining. The protocols for GUS staining and section analysis have been described previously ${ }^{51}$. To determine OsNPF2.2 expression under normal $\mathrm{N}$ conditions, plants were grown in a paddy field under normal fertilizer application. For nitrate induction, the rice seedlings were grown in IRRI solution with $1.4 \mathrm{mM}$ $\mathrm{KNO}_{3}$ for $10 \mathrm{~d}$ and then transferred to IRRI solution with $1.4 \mathrm{mM} \mathrm{NH}_{4} \mathrm{SO}_{4}$ as the $\mathrm{N}$ source for $\mathrm{NO}_{3}$ - deprivation for $1 \mathrm{~d}$. Finally, the plants were transferred back into IRRI solution with $10 \mathrm{mM} \mathrm{KNO}_{3}$ to induce GUS activity within $2 \mathrm{~h}$.

Semi section and transmission electron microscopy analysis. Sample preparation was carried out according to the procedures described by Park et al. ${ }^{57}$, Ultra-sections were examined under transmission electron microscopy (JEM-1010 electron microscope; JEOL, Tokyo, Japan). For semi section observation, the $2 \mu \mathrm{m}$ thick sections were stained with $0.5 \%$ toluidine blue and then observed and photographed using a fluorescence microscope (AXIOPLAN 2; Zeiss, Jena, Gemany).

Transient expression of $O s N P F 2.2$ in rice protoplasts. To determine the subcellular localization of OsNPF2.2, $p 35 S-O s N P F 2.2-G F P$ and $p 35 S-G F P$ were introduced into rice protoplasts. The protocols for rice protoplast preparation and transformation have previously been described ${ }^{58}$. Protoplasts were observed using a confocal laserscanning microscope (Leica TCS 5 SP5 AOBS), a Leica Microsystem LAS AF, or a Leica DMI6000B inverted fluorescence microscope, as previously described ${ }^{40}$.

Nitrate content analysis by high-performance liquid chromatography. Rice xylem sap collection was performed according to the protocol described by Tang et al $l^{59}$, as follows. The rice plants were grown in IRRI solution containing $1.4 \mathrm{mM} \mathrm{NH}_{4} \mathrm{NO}_{3}$ for 8 weeks; then, seedlings were transferred to IRRI solution containing $1.4 \mathrm{mM}$ $\mathrm{NH}_{4} \mathrm{SO}_{4}$ as the $\mathrm{N}$ source for $\mathrm{NO}_{3}{ }^{-}$deprivation for 1 week. Next, the plants were cut $4 \mathrm{~cm}$ above the ground level, the roots were immediately transferred to $10 \mathrm{mMNO}_{3}{ }^{-}$, and a preweighed absorbent cotton ball used to collect the xylem exudate was attached to the cut surface and covered with plastic film for $2 \mathrm{~h}$. Next, the collected xylem sap was squeezed from the cotton by using a syringe, and the volume of the exudate was calculated based on the increase in the weight of the cotton. For extraction of total nitrate, the roots and shoots were collected separately, and the nitrate was extracted in boiling distilled $\mathrm{H}_{2} \mathrm{O}$ for $30 \mathrm{~min}$. Nitrate concentration was determined by highperformance liquid chromatography ${ }^{60}$ using a PARTISIT 10 SAX (strong anion exchanger) column (Waterman), with $50 \mathrm{mM} \mathrm{KH}_{2} \mathrm{PO}_{4}$ buffer, $\mathrm{pH}$ 3.0, as the mobile phase.

1. Wang, Y., Hsu, P. K. \& Tsay, Y. Uptake, allocation and signaling of nitrate. Trends Plant Sci. 17, 458-467 (2012).

2. Xu, G., Fan, X. \& Miller, A. J. Plant nitrogen assimilation and use efficiency. Annu. Rev. Plant Biol. 63, 153-182 (2012).

3. Kronzucker, H. J., Siddiqi, M. Y., Glass, A. D. \& Kirk, G. J. Nitrate-ammonium synergism in rice. A subcellular flux analysis. Plant Physiol. 119, 1041-1046 (1999).

4. Kronzucker, H. J., Glass, A. D. M., Siddiqi, M. Y. \& Kirk, G. J. D. Comparative kinetic analysis of ammonium and nitrate acquisition by tropical lowland rice: implications for rice cultivation and yield potential. New Phytol. 145, 471-476 (2000).

5. Shi, Y., Shen, Q., Mao, Z. \& Xu, G. Time and horizontal spatial variations of $\mathrm{NH}_{4}{ }^{+}$ $\mathrm{N}$ and $\mathrm{NO}_{3}{ }^{-}-\mathrm{N}$ of rhizospheric soil with rice cultivation on upland condition mulched with half-decomposed rice straw. Scientia Agricultura Sinica 35, 520-524 (2002).

6. Dechorgnat, J. et al. From the soil to the seeds: the long journey of nitrate in plants. J. Exp. Bot. 62, 1349-1359 (2011).

7. Miller, A. J., Fan, X., Orsel, M., Smith, S. J. \& Wells, D. M. Nitrate transport and signalling. J. Exp. Bot. 58, 2297-2306 (2007).

8. Krapp, A. et al. Nitrate transport and signalling in Arabidopsis. J. Exp. Bot. 65, 789-798 (2014)

9. Sasaki, T. et al. Closing plant stomata requires a homolog of an aluminumactivated malate transporter. Plant Cell Physiol. 51, 354-365 (2010).

10. Léran, S. et al. A unified nomenclature of NITRATE TRANSPORTER 1/ PEPTIDE TRANSPORTER family members in plants. Trends Plant Sci. 19, 5-9 (2014).

11. Nour-Eldin, H. H. et al. NRT/PTR transporters are essential for translocation of glucosinolate defence compounds to seeds. Nature 488, 531-534 (2012).

12. Kanno, Y. et al. Identification of an abscisic acid transporter by functional screening using the receptor complex as a sensor. Proc. Natl. Acad. Sci. USA 109, 9653-9658 (2012).

13. Krouk, G. et al. Nitrate-regulated auxin transport by NRT1.1 defines a mechanism for nutrient sensing in plants. Dev. Cell 18, 927-937 (2010). 
14. Liu, K., Huang, C. \& Tsay, Y. CHL1 is a dual-affinity nitrate transporter of Arabidopsis involved in multiple phases of nitrate uptake. Plant Cell 11, 865-874 (1999).

15. Wang, R., Liu, D. \& Crawford, N. M. The Arabidopsis CHL1 protein plays a major role in high-affinity nitrate uptake. Proc. Natl. Acad. Sci. USA 95, 15134-15139 (1998)

16. Morere-Le Paven, M. C. et al. Characterization of a dual-affinity nitrate transporter MtNRT1.3 in the model legume Medicago truncatula. J. Exp. Bot. 62, 5595-5605 (2011).

17. Tsay, Y., Schroeder, J. I., Feldmann, K. A. \& Crawford, N. M. The herbicide sensitivity gene CHL1 of Arabidopsis encodes a nitrate-inducible nitrate transporter. Cell 72, 705-713 (1993).

18. Léran, S., Munos, S., Brachet, C., Tillard, P., Gojon, A. \& Lacombe, B. Arabidopsis NRT1.1 is a bidirectional transporter involved in root-to-shoot nitrate translocation. Mol. Plant 6, 1984-1987 (2013).

19. Lin, S. et al. Mutation of the Arabidopsis NRT1.5 nitrate transporter causes defective root-to-shoot nitrate transport. Plant Cell 20, 2514-2528 (2008).

20. Li, J. Y. et al. The Arabidopsis nitrate transporter NRT1.8 functions in nitrate removal from the xylem sap and mediates cadmium tolerance. Plant Cell 22, 1633-1646 (2010).

21. Wang, Y. \& Tsay, Y. Arabidopsis nitrate transporter NRT1.9 is important in phloem nitrate transport. Plant Cell 23, 1945-1957 (2011).

22. Chiu, C., Lin, C., Hsia, A., Su, R., Lin, H. \& Tsay, Y. Mutation of a nitrate transporter, AtNRT1:4, results in a reduced petiole nitrate content and altered leaf development. Plant Cell Physiol. 45, 1139-1148 (2004).

23. Fan, S., Lin, C., Hsu, P., Lin, S. \& Tsay, Y. The Arabidopsis nitrate transporter NRT1.7, expressed in phloem, is responsible for source-to-sink remobilization of nitrate. Plant Cell 21, 2750-2761 (2009).

24. Almagro, A., Lin, S. \& Tsay, Y. Characterization of the Arabidopsis nitrate transporter NRT1.6 reveals a role of nitrate in early embryo development. Plant Cell 20, 3289-3299 (2008).

25. Wang, R., Xing, X., Wang, Y., Tran, A. \& Crawford, N. M. A genetic screen for nitrate regulatory mutants captures the nitrate transporter gene NRT1.1. Plant Physiol. 151, 472-478 (2009).

26. Remans, T. et al. The Arabidopsis NRT1.1 transporter participates in the signaling pathway triggering root colonization of nitrate-rich patches. P. Natl. Acad. Sci. USA 103, 19206-19211 (2006).

27. Garnett, T., Conn, V. \& Kaiser, B. N. Root based approaches to improving nitrogen use efficiency in plants. Plant Cell Environ. 32, 1272-1283 (2009)

28. Remans, T., et al. A central role for the nitrate transporter NRT2.1 in the integrated morphological and physiological responses of the root system to nitrogen limitation in Arabidopsis. Plant Physiol. 140, 909-921 (2006).

29. Fan, M., et al. Improving crop productivity and resource use efficiency to ensure food security and environmental quality in China. J. Exp. Bot. 63, 13-24 (2012).

30. Sun, H. et al. Heterotrimeric $\mathrm{G}$ proteins regulate nitrogen-use efficiency in rice. Nat. Genet. 46, 652-656 (2014).

31. Tsay, Y., Chiu, C., Tsai, C., Ho, C. \& Hsu, P. Nitrate transporters and peptide transporters. FEBS Lett. 581, 2290-2300 (2007).

32. Zhao, X., Huang, J., Yu, H., Wang, L. \& Xie, W. Genomic survey, characterization and expression profile analysis of the peptide transporter family in rice (Oryza sativa L.). BMC Plant Biol. 10, 92 (2010).

33. Araki, R. \& Hasegawa, H. Expression of rice (Oryza sativa L.) genes involved in high-affinity nitrate transport during the period of nitrate induction. Breeding Sci. 56, 295-302 (2006).

34. Cai, C. et al. Gene structure and expression of the high-affinity nitrate transport system in rice roots. J. Integr. Plant Bio. 50, 443-451 (2008).

35. Lin, C., Koh, S., Stacey, G., Yu, S., Lin, T. \& Tsay, Y. Cloning and functional characterization of a constitutively expressed nitrate transporter gene, OsNRT1, from rice. Plant Physiol. 122, 379-388 (2000).

36. Xia, X. et al. Rice nitrate transporter OsNPF2.4 functions in low-affinity acquisition and long-distance transport. J. Exp. Bot. 66, 317-331 (2015).

37. Chiang, C., Stacey, G. \& Tsay, Y. Mechanisms and functional properties of İtwo peptide transporters, AtPTR2 and fPTR2. J. Biol. Chem. 279, 30150-30157 (2004)

38. Stacey, G., Koh, S., Granger, C. \& Becker, J. Peptide transport in plants. Trends Plant Sci. 7, 257-263 (2002).

39. Odell, J., Nagy, F. \& Chua, N. Identification of dna-sequences required for activity of the cauliflower mosaic virus-35s promoter. Nature 313, 810-812 (1985).

40. Nelson, B., Cai, X. \& Nebenfuhr, A. A multicolored set of in vivo organelle markers for co-localization studies in Arabidopsis and other plants. Plant J. 51, 1126-1136 (2007).

41. Jeong, D. H. et al. T-DNA insertional mutagenesis for activation tagging in rice. Plant Physiol. 130, 1636-1644 (2002)

42. Yi, J. \& An, G. Utilization of T-DNA tagging lines in rice. J. Plant Biol. 56, 85-90 (2013).
43. Schroeder, J. et al. Using membrane transporters to improve crops for sustainable food production. Nature 497, 60-66 (2013).

44. Ouyang, J., Cai, Z., Xia, K., Wang, Y., Duan, J. \& Zhang, M. Identification and analysis of eight peptide transporte rhomologs in rice. Plant Sci. 179, 374-382 (2010).

45. Andrews, $M$. The partitioning of nitrate assimilation between root and shoot of higher-plants. Plant Cell Environ. 9, 511-519 (1986)

46. Puig, J., Pauluzzi, G., Guiderdoni, E. \& Gantet, P. Regulation of shoot and root development through mutual signaling. Mol. Plant 5, 974-983 (2012).

47. Yan, M., Fan, X., Feng, H., Miller, A. J., Shen, Q. \& Xu, G. Rice OsNAR2.1 interacts with OsNRT2.1, OsNRT2.2 and OsNRT2.3a nitrate transporters to provide uptake over high and low concentration ranges. Plant Cell Physiol. 34, 1360-1372 (2011).

48. Eckardt, N. A. Nitrogen-sparing mechanisms in Chlamydomonas: reduce, reuse, recycle, and reallocate. Plant Cell 26, 1379 (2014).

49. Schmollinger, S. et al. Nitrogen-sparing mechanisms in Chlamydomonas affect the transcriptome, the proteome, and photosynthetic metabolism. Plant Cell 26, 1410-1435 (2014).

50. Li, S. et al. Short panicle1 encodes a putative PTR family transporter and determines rice panicle size. Plant J. 58, 592-605 (2009).

51. Fang, Z. et al. Altered expression of the PTR/NRT1 homologue OsPTR9 affects nitrogen utilization efficiency, growth and grain yield in rice. Plant Biotechnol. J. 11, 446-458 (2013)

52. Alboresi, A., Gestin, C., Leydecker, M. T., Bedu, M., Meyer, C. \& Truong, H. N Nitrate, a signal relieving seed dormancy in Arabidopsis. Plant Cell Environ. 28, 500-512 (2005)

53. Reggiani, R., Mattana, M., Aurisano, N. \& Bertani, A. Utilization of stored nitrate during the anaerobic germination of rice seeds. Plant Cell Physiol. 34, 379-383 (1993).

54. Chopin, F. et al. The Arabidopsis ATNRT2.7 nitrate transporter controls nitrate content in seeds. Plant Cell 19, 1590-1602 (2007).

55. Yoshida, S., Forno, D. A., Cook, J. H. \& Gomez, K. A. Routine procedures for growing rice plants in culture solution. In Laboratory Manual for Physiological Studies of Rice (Yoshida, S., Forno, D. A., Cook, J. H., and Gomez, K. A. eds): International Rice Research Institute, Los Banos, Philippines., pp. 61-66 (1976).

56. Wang, Z. et al. A practical vector for efficient knockdown of gene expression in rice (Oryza sativa L.). Plant Mol. Biol. Rep. 22, 409-417 (2004).

57. Park, J. J. et al. Mutation in wilted dwarf and lethal 1(WDL1) causes abnormal cuticle formation and rapid water loss in rice. Plant Mol. Biol. 74, 91-103 (2010).

58. Zhang, Y. et al. A highly efficient rice green tissue protoplast system for transient gene expression and studying light/chloroplast-related processes. Plant Methods 7, 30 (2011).

59. Tang, Z. et al. Knockdown of a rice stelar nitrate transporter alters long-distance translocation but not root influx. Plant Physiol. 160, 2052-2063(2012).

60. Thayer, J. R. \& Huffaker, R. C. Determination of nitrate and nitrite by highpressure liquid chromatography: comparison with other methods for nitrate determination. Anal. Biochem. 102, 110-119 (1980).

\section{Acknowledgments}

This research was supported by grants (31272240/S2013020012830) of NSFC/GD NSF, grant (KSCX3-EW-N-07) of CAS, the united fund of SCBG and IPPE, and K.C. Wong Education Foundation.

\section{Author contributions}

Y.L. and M.Z. designed the experiments and wrote the manuscript. Y.L., O., Y.Y.W., R.H. and K.X. performed the experiments. J.D., Y.W., Y.F.T. and M.Z. improved the manuscript.

\section{Additional information}

Supplementary information accompanies this paper at http://www.nature.com/ scientificreports

Competing financial interests: The authors declare no competing financial interests. How to cite this article: Li, Y. et al. Disruption of the rice nitrate transporter OsNPF2.2 hinders root-to-shoot nitrate transport and vascular development. Sci. Rep. 5, 9635; DOI:10.1038/srep09635 (2015)

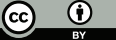

This work is licensed under a Creative Commons Attribution 4.0 International License. The images or other third party material in this article are included in the article's Creative Commons license, unless indicated otherwise in the credit line; if the material is not included under the Creative Commons license, users will need to obtain permission from the license holder in order to reproduce the material. To view a copy of this license, visit http://creativecommons.org/licenses/by/4.0/ 